\title{
A CHARACTERIZATION OF SEMISPAN OF CONTINUA
}

\author{
EDWIN DUDA
}

\begin{abstract}
The main results of this paper are characterizations of semispan $\varepsilon, \varepsilon \geqslant 0$, for those metric spaces which are atriodic hereditarily unicoherent continua. The results follow from Theorem 1 which gives conditions under which the union of two continua, each of semispan less than or equal to $\varepsilon$, has semispan less than or equal to $\varepsilon$.
\end{abstract}

1. Introduction. The notion of span of a compact metric space and its natural generalization semispan were defined by A. Lelek in 1964 and 1977 respectively [6, 7]. It follows from the definitions that semispan is greater than or equal to span and both functions are monotone with respect to closed subsets. It can be shown directly that a nonunicoherent continuum or a triod have span greater than zero. All chainable continua have semispan zero and those continua without the fixed point property have span greater than zero. W. T. Ingram constructed an example of an atriodic hereditarily unicoherent continuum with span greater than zero [5]. The author and J. Kell have shown that if an atriodic, hereditarily unicoherent continuum is the sum of two continua of semispan zero, then the continuum itself has semispan zero [3]. J. F. Davis has shown for a metric continuum in which every subcontinuum is in class $W$, span and semispan are equal for all subcontinua [1]. In particular, for continua, semispan zero and span zero are equivalent. M. Cuervo and E. Duda have shown that a hereditarily unicoherent atriodic metric continuum has span zero if and only if each indecomposable subcontinuum has span zero [2]. In this note we propose to demonstrate that a hereditarily unicoherent atriodic metric continuum has semispan $\varepsilon, \varepsilon \geqslant 0$, if and only if it has an indecomposable subcontinuum which has semispan $\varepsilon$ irreducibly and has no indecomposable subcontinuum with semispan greater than $\varepsilon$.

2. Notations and definitions. A continuum is a compact connected metric space. Let $\pi_{1}$ and $\pi_{2}$ represent the natural projection mappings of the Cartesian Product $X \times X$ onto $X$. For a set $D$, represent the closure of $D$ by $\bar{D}$, the interior of $D$ by int $D$ and the frontier or boundary of $D$ by $\operatorname{Fr} D$. The semispan of a continuum $(X, \rho), \sigma_{0}(X)$ is the least upper bound of the set of real numbers $\varepsilon$ such that there is a continuum $Z_{\varepsilon}$ in $X \times X$ with the property that $\pi_{2}\left(Z_{\varepsilon}\right) \subset \pi_{1}\left(Z_{\varepsilon}\right)$ and $\rho(x, y) \geqslant \varepsilon$ for all $(x, y)$ in $Z_{\varepsilon}$. The number obtained by additionally requiring $\pi_{2}\left(Z_{\varepsilon}\right)=\pi_{1}\left(Z_{\varepsilon}\right)$ is called the span of $X$ and is denoted by $\sigma(X)$. A continuum is atriodic provided it contains no triod and is hereditarily unicoherent provided each subcontinuum is

Received by the editors September 10, 1984 and, in revised form, March 7, 1985.

1980 Mathematics Subject Classification. Primary 54F20, 54C10; Secondary 54F50, 54H25.

Key words and phrases. Span, semispan.

(C) 1986 American Mathematical Society $0002-9939 / 86 \$ 1.00+\$ .25$ per page 
unicoherent. A continuum is indecomposable if it cannot be represented as the union of two proper subcontinua. In an atriodic hereditarily unicoherent continuum we define for each $x \in X$ the continuum $K_{x}=\bigcap\{K \mid K$ a subcontinuum of $X$ and $x \in$ int $K\}$. The properties of $K_{x}$ are thoroughly outlined in [2].

3. Main theorems. Suppose $X$ is an atriodic metric continuum. In [3] it was shown that if $X=A \cup B$ and $\sigma(A)=\sigma(B)=0$, where $A, B$, and $A \cap B$ are continua, then $\sigma(X)=0$. In [4] it was shown that if $X=A \cup B$, where $A$ and $B$ are continua with $\sigma_{0}(A) \leqslant \varepsilon, \sigma_{0}(B) \leqslant \varepsilon, \varepsilon \geqslant 0, X$ is hereditarily unicoherent, and $A \cap B$ is a continuum of continuity, then $\sigma_{0}(X) \leqslant \varepsilon$. The next theorem generalizes both of the results quoted above. The proof is an extension of the proof in [3] and we include a complete proof.

THEOREM 1. If $X$ is an atriodic hereditarily unicoherent continuum and $X=A \cup B$, where $A$ and $B$ are continua with $\sigma_{0}(A) \leqslant \varepsilon$ and $\sigma_{0}(B) \leqslant \varepsilon, \varepsilon \geqslant 0$, then $\sigma_{0}(X) \leqslant \varepsilon$.

Proof. Let $X=A \cup B$, where $A$ and $B$ are as in the theorem. Let $H=A \cap B$ and note that we may assume $\overline{A-B}=A$ and $\overline{B-A}=B$. If the semispan of $X$ is greater than $\varepsilon$, there exists a continuum $Z$ contained in $X \times X$ whose distance from the diagonal of $X \times X$ is greater than $\varepsilon$. We can make the following assumptions: (i) $\pi_{2}(Z) \subset \pi_{1}(Z)$, (ii) $\pi_{1}(Z)$ meets $A-B$ and $B-A$ (otherwise $Z$ would be contained in $A \times A$ or $B \times B$, (iii) $\pi_{1}(Z)=X$.

We first argue that $Z$ meets the interiors of $A \times B$ and $B \times A$. Suppose that $Z$ misses the int $(A \times B)$. Then $\pi_{1}(Z \cap(A \times A)) \supset A-B$. Let $\left\{C_{\alpha}\right\}$ be the collection of components of $Z-(B \times X)$. Thus $\bar{C}_{\alpha} \cap(H \times X) \neq \varnothing$ for all $\alpha$ and hence $\pi_{1}\left(\bar{C}_{\alpha}\right) \cap H \neq \varnothing$ for all $\alpha$. Since $X$ is atriodic, $\pi_{1}\left(C_{\alpha}\right) \subset \pi_{1}\left(C_{\beta}\right)$ or $\pi_{1}\left(C_{\beta}\right) \subset \pi_{1}\left(C_{\alpha}\right)$ for all $\alpha$ and $\beta$. This and the fact that $A-B \subset \pi_{1}\left(\cup C_{\alpha}\right)$ imply that there exists a continuum $C$ contained in $Z \cap(A \times A)$ with $A-B \subset \pi_{1}(C)$. Consequently $\pi_{1}(C)$ $=A$ and this in turn implies $\sigma_{0}(A)>\varepsilon$. Since this is not possible, $Z$ must meet the $\operatorname{int}(A \times B)$ and similarly $Z$ must meet the $\operatorname{int}(B \times A)$.

Let $\left(a_{1}, b_{1}\right) \in \operatorname{int}(A \times B) \cap Z$ and $\left(b_{2}, a_{2}\right) \in \operatorname{int}(B \times A) \cap Z$. Further let $U_{1}, U_{2}, \ldots$ be a sequence of open sets such that: (i) $\bar{U}_{i+1} \subset U_{i}$ for all $i$, (ii) $H=\bigcap_{i=1}^{\infty} U_{i}$, (iii) $a_{1}, a_{2}, b_{1}$, and $b_{2}$ are not in $\bar{U}_{1}$. Set $E_{i}=A \times\left(B-U_{i}\right)$ and $F_{i}=B \times\left(A-U_{i}\right)$. It follows that $\left(a_{1}, b_{1}\right) \in E_{i}$ and $\left(b_{2}, a_{2}\right) \in F_{i}$ for all $i$. Furthermore $E_{i}$ and $F_{i}$ are disjoint closed sets for each $i$. Since $Z$ is connected, there is a component $C_{i}$ of $Z-\left(E_{i} \cup F_{i}\right)$ whose closure meets $E_{i}$ and $F_{i}$. Note that $\pi_{2}\left(C_{i}\right)$ meets $A-B$ and $B-A$ for each $i$ and hence $\pi_{2}\left(C_{i}\right) \supset H$ for all $i$. The remainder of the proof reduces to two cases: (i) $\pi_{1}\left(\bar{C}_{i}\right)$ meets $A-B$ and $B-A$ for all $i$, (ii) $\pi_{1}\left(\bar{C}_{i}\right)$ is contained in $A$ or $\dot{B}$ for some $i$.

Case (i). If $\pi_{1}\left(\bar{C}_{i}\right)$ meets $A-B$ and $B-A$ for all $i$, then for each $i$ there exists an integer $N_{i}$ such that for $j \geqslant N_{i}$ there is a component $L_{i j}$ of $\bar{C}_{i} \cap\left(U_{j} \times X\right)$ whose closure meets $\left(\operatorname{Fr}\left(U_{j}\right) \cap A\right) \times X$ and $\left(\operatorname{Fr}\left(U_{j}\right) \cap B\right) \times X$. Obviously $\pi_{1}\left(\bar{L}_{i j}\right) \supset H$ for each $j \geqslant N_{i}$. Some subsequence of $\left\{\bar{L}_{i j}\right\}, j \geqslant N_{i}$, converges to a continuum $L_{i}$ contained in $(H \times X) \cap Z-\left(E_{i} \cup F_{i}\right)$. Since $H \subset \pi_{1}\left(\bar{L}_{i j}\right)$ for all $j \geqslant N_{j}$, it follows 
that $H \subset \pi_{1}\left(L_{i}\right)$. The sequence $\left\{L_{i}\right\}$ has a subsequence which converges to a continuum $L$ contained in $(H \times H) \cap Z$ and clearly $\pi_{1}(L)=H$ and $\pi_{2}(L) \subset H$. Thus $\pi_{1}(L) \supset \pi_{2}(L)$ and this implies $\sigma_{0}(H)>\varepsilon$ which is not possible.

Case (ii). We can assume that $\pi_{1}\left(\bar{C}_{i}\right)$ is contained in $A$ for some $i$. Thus $\bar{C}_{i}$ is contained in $A \times X$ and meets $H \times(A-H)$ so these three sets have a point $(h, a)$ in common. For $j \geqslant i$ the closure of the component $D_{j}$ of $\bar{C}_{i}-E_{j}$ containing $(h, a)$ meets $E_{j}$. Hence $H \subset \pi_{2}\left(\bar{D}_{j}\right)$ for each $j \geqslant i$ and so, $H \subset \pi_{2}\left(\bigcap_{j \geqslant i} \bar{D}_{j}\right)$. Let $D=$ $\cap_{j \geqslant i} \bar{D}_{j}$, then $(h, a) \in D$ and $D$ is contained in $A \times A$. We can now assume $H-\pi_{1}(D) \neq \varnothing$ and $\pi_{2}(D)-H$ is a proper subset of $\pi_{1}(D)-H$, otherwise in either case $D$ would have its projection in one direction contained in the projection of $D$ in the other direction. Let $H-\pi_{1}(D)=\bigcup_{i=1}^{\infty} M_{i}$, where $M_{i}$ is compact and $M_{i} \subset$ int $M_{i+1} \neq \varnothing$ (relative to $H$ ) for all $i$. Let $L_{i}$ be the closure of the component of $D-\left(A \times M_{i}\right)$ which contains $(h, a)$. Then $\pi_{2}\left(L_{i}\right) \cap M_{i} \neq \varnothing$, so $\pi_{2}\left(L_{i}\right)$ contains a point of $H$ not in $\pi_{1}(D)$. If $\pi_{2}\left(L_{i}\right)$ does not contain $\pi_{1}(D) \cap H$, then $\pi_{2}\left(L_{i}\right)-H$ is a connected open set whose closure does not contain $\pi_{1}(D) \cap H$ (otherwise

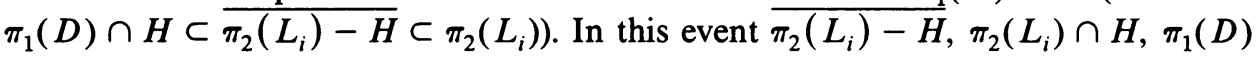
$\cap H$ determine a triod so it must be the case that $\pi_{2}\left(L_{i}\right) \cap H \supset \pi_{1}(D) \cap H$.

Let $N_{1}$ be the limit of a convergent subsequence of the $L_{i}$ and notice that $\pi_{2}\left(N_{1}\right) \cap H=\pi_{1}(D) \cap H \supset \pi_{1}\left(N_{1}\right) \cap H$ and that $(h, a) \in N_{1}$. Now if $\pi_{2}\left(N_{1}\right) \cap H$ $=\pi_{1}\left(N_{1}\right) \cap H$ or $\pi_{2}\left(N_{1}\right)-H \supset \pi_{1}\left(N_{1}\right)-H$, then $N_{1}$ is a continuum in $A \times A$ with the required projections to show $\sigma_{0}(A)>\varepsilon$. Thus we can assume $\pi_{1}\left(N_{1}\right) \cap H$ is a proper subcontinuum of $\pi_{2}\left(N_{1}\right) \cap H$ and $\pi_{1}\left(N_{1}\right)-H$ properly contains $\pi_{2}\left(N_{1}\right)-$ $H$.

By taking $\pi_{2}\left(N_{1}\right) \cap H-\pi_{1}\left(N_{1}\right) \cap H=\bigcup_{i=1}^{\infty} M_{i}$, where $M_{1}$ is compact and $M_{i} \subset$ int $M_{i+1} \neq \varnothing$ (relative to $\pi_{2}\left(N_{1}\right) \cap H$ ), a new sequence of continua $L_{i}$ can be constructed with the properties that $(h, a) \in L_{i}$ for all $i$ and $\pi_{2}\left(L_{i}\right) \cap H \supset \pi_{1}\left(N_{1}\right)$ $\cap H$. Let $N_{2}$ be a continuum which is the limit of a convergent subsequence of the $L_{i}$. Then $(h, a) \in N_{2}$ and $\pi_{2}\left(N_{2}\right) \cap H=\pi_{1}\left(N_{1}\right) \cap H \supset \pi_{1}\left(N_{2}\right) \cap H$. Now, as before, if $\pi_{2}\left(N_{2}\right) \cap H=\pi_{1}\left(N_{2}\right) \cap H$ or $\pi_{2}\left(N_{2}\right)-H \supset \pi_{1}\left(N_{2}\right)-H$, then $N_{2}$ is the required continuum.

We can repeat the construction above until for some $m, N_{m}$ is the required continuum or we obtain an infinite sequence $\left\{N_{m}\right\}$ with the following properties. The point $(h, a) \in N_{m}$ for all $m$ and $\pi_{2}\left(N_{m+1}\right) \cap H=\pi_{1}\left(N_{m}\right) \cap H$ for all $m$. If $N$ is the limit of a convergent subsequence of the sequence $\left\{N_{m}\right\}$, then $(h, a) \in N$ and $\pi_{2}(N) \cap H=\pi_{1}(N) \cap H$ and thus $N$ is the required continuum.

THEOREM 2. Let $X$ be an atriodic hereditarily unicoherent continuum with semispan $\varepsilon$. Then $X$ contains an indecomposable subcontinuum $I$ which has semispan $\varepsilon$ irreducibly.

Proof. Since $\sigma_{0}(X)=\varepsilon$, it follows by the Brouwer Reduction theorem that $X$ contains a subcontinuum $I$ which has semispan $\varepsilon$ and each proper subcontinuum of $I$ has semispan less than $\varepsilon$. By Theorem $1, I$ must be indecomposable.

THEOREM 3. Let $X$ be an atriodic hereditarily unicoherent continuum. Then $\sigma_{0}\left(K_{x}\right)$ $\leqslant \varepsilon$ for all $x$ in $X$ if and only if $\sigma_{0}(I) \leqslant \varepsilon$ for each indecomposable subcontinuum $I$ of $X$. 
Proof. Suppose $\sigma_{0}\left(K_{x}\right) \leqslant \varepsilon$ for all $x \in X$. Let $I$ be an indecomposable subcontinuum of $X$ and $y \in I$. Clearly $I \subset K_{y}$ and by the monotone property of $\sigma_{0}$ we have $\sigma_{0}(I) \leqslant \sigma_{0}\left(K_{y}\right) \leqslant \varepsilon$.

Suppose now each indecomposable subcontinuum has semispan less than or equal to $\varepsilon$. If $x \in X$ and $\sigma_{0}\left(K_{x}\right)>\varepsilon$, then by Theorem $1, K_{x}$ would contain an indecomposable subcontinuum $I$ with $\sigma_{0}(I)>\varepsilon$. Since this is not possible, $\sigma_{0}\left(K_{x}\right) \leqslant \varepsilon$ for all $x \in X$.

COROLLARY 1. The following are equivalent for an atriodic hereditarily unicoherent continuum:

(i) $\sigma_{0}(X) \leqslant \varepsilon$;

(ii) $\sigma_{0}\left(K_{x}\right) \leqslant \varepsilon$ for all $x \in X$;

(iii) $\sigma_{0}(I) \leqslant \varepsilon$ for all indecomposable subcontinua $I$ of $X$.

\section{REFERENCES}

1. James F. Davis, The equivalence of zero span and zero semispan, Proc. Amer. Math. Soc. 90 (1984), 133-138.

2. M. Cuervo and E. Duda, A characterization of span zero, Houston J. Math. (to appear).

3. E. Duda and J. Kell III, Two sum theorems for semispan, Houston J. Math. 8 (1982), 317-321.

4. E. Duda, A sum theorem for semispan of continua, Proc. of the Fifth Prague Topology Symposium (1981), Heldermann Verlag, Berlin, 1982, pp. 162-163.

5. W. T. Ingram, An atriodic tree-like continuum with positive span, Fund. Math. 77 (1972), 99-107.

6. A. Lelek, Disjoint mappings and the span of spaces, Fund. Math. 55 (1964), 199-214.

7. __ On surjective span and semispan of connected metric spaces, Colloq. Math. 37 (1977), 34-35.

Department of Mathematics, University of Miami, Coral Gables, Florida 33124 